\title{
Combined use of active and passive surface waves for the $S$-wave velocity structure delineation beneath the Balikun Basin, China
}

\author{
Jing Lin*, Kaifeng Zhao, Yinhe Luo, Hongri Xu \\ Hubei Subsurface Multi-scale Imaging Key Laboratory, \\ Institute of Geophysics and Geomatics, China University of Geosciences, (CUG) \\ Wuhan, Hubei, 430074, China. \\ E-mail: lj_207@126.com
}

\begin{abstract}
Rayleigh waves have been widely used to estimate the shear-wave velocity structure in the fields of shallow engineering and environmental detection. In this study, active and passive surface wave surveys were performed sequentially on the same survey line across the eastern Balikun Basin. MASW (Multi-channel Analysis of Surface waves) technology was utilized for active sources to obtain the phase velocity dispersion curves of the fundamental-mode Rayleigh waves. For the passive surface wave surveys, cross correlations were performed to retrieve the empirical Green's functions, from which the phase velocity dispersion curves can be measured by FTAN (Frequency-Time Analysis) method. We applied the 1D Surface wave tomography (SWT) to construct a phase velocity map which combines the two sets of dispersion curves obtained from active and passive Rayleigh waves. Finally, the obtained phase velocity dispersion curves were inverted for $1 \mathrm{D}$ shear velocity profiles and then 2D Vs profiles was constructed by assembling these 1D profiles. The results show that the investigation depth of active surface wave measurement is limited to several decameters, while the investigation depth of passive surface wave measurement can reach over a hundred meters, and combining these two methods can achieve a greater maximum depth of the Vs profile without sacrificing the near-surface resolution.
\end{abstract}

Keywords-active sources; passive sources; rayleigh waves; swave velocity structure; balikun basion

\section{INTRODUCTION}

As the surface wave method has received wide attention in recent years as one of the popular methods to detect the shear-wave velocity of near-surface materials, it has been applied in various fields such as geotechnical engineering and environment monitoring. According to the types of seismic source, the Rayleigh wave exploration can be divided into active and passive methods. The active method using an artificial seismic source, like a sledgehammer, can usually estimate the shear-wave velocity(Vs) structure down to several tens of meters, however, the investigation depth is insufficient to meet the requirements of certain projects. Although enhancing the power of the active source can achieve the goal of deeper Vs structure, the gained depth range is often insignificant with a heavy-weight dropper, while morepowerful active source such as explosive source will pollute and devastate the environment, which is inapplicable in most fields especially in the cities. Instead, the passive surface waves generated by cultural activities (e.g., traffic, mechanical vibration, etc.) have longer wavelengths than active ones, which make it possible to gain the information from deeper layers. The frequency range of the dispersion curve extracted from active survey is relatively high (e.g., 10 40Hz), While the passive survey can fill the dispersion trend at lower frequencies (for e.g., $1 \sim 10 \mathrm{~Hz}$ ). So combined these two sets of dispersion curves can achieve a Vs profile for a wide investigation depth.

The Multi-channel Analysis of Surface Waves (MASW) method is the most widely-used active method to estimate the near-surface Vs structure nowadays, which records the signal with multiple receivers and performs a simultaneous analysis of multiple geophone records through transformation from time-space domains to frequency-velocity domains ${ }^{[1,2]}$. During 2000 to 2008, a large amount of research and comparison experiments have been performed to improve the MASW method, decreasing the execution time and increasing the accuracy of dispersion curves when compared to the earlier Spectral Analysis of Surface Waves (SASW) approach. Then the variant of MASW has also been used to passive tests by using linear arrays alongside a road to identify the dispersion curves successfully ${ }^{[3]}$. Different from the Multichannel methods, the Frequency-Time Analysis (FTAN) method can be applied in a setting with only a single channel to measure group velocity even when there is higher mode contamination. When the source is known, the phase velocity can also be measured. The FTAN is successful used in both seismology and seismic engineering ${ }^{[4-6]}$. In order to improve the investigation depth of the active MASW, Park and Miller (2005) employed the passive and active versions of the multichannel analysis of surface waves (MASW) method for an increased investigation depth ${ }^{[7]}$. The two-source combined method has also been applied and achieved satisfied results at home ${ }^{[8,9]}$.

In this paper, we performed linear active and passive surface wave surveys at the same fields inside the Balikun Basin, The dispersion curves of fundamental-mode Rayleigh wave were extracted through High Resolution Linear Radon Transform (HRLRT ${ }^{[10]}$ ) for active survey and FTAN ${ }^{[5]}$ for passive survey, respectively. With combining these two sets of 
dispersion curves by 1D Surface Wave Tomography (SWT), more broadband dispersion curves have been achieved to invert Vs structure for a deep range.

\section{METHODS}

Both of active and passive Rayleigh wave exploration generally include three steps: (1) recording the active (or passive) signals which carry the information of Rayleigh surface waves; (2) extracting the dispersion curves from active multichannel surface-wave records (or empirical Green's functions obtained from the cross-correlations of two-station noise records); (3) Obtaining Vs structure by inversion of the phase velocity dispersion curves of fundamental-mode Rayleigh waves adopting an iterative linearized least-square inversion scheme.

Combining the active and passive surface wave methods represents putting dispersion curves extracted from active survey $(10 \sim 30 \mathrm{~Hz})$ and passive survey $(2 \sim 10 \mathrm{~Hz})$ together to compose broadband dispersion curves $(2 \sim 30 \mathrm{~Hz})$ which can be used to invert the Vs profile of $\sim 200 \mathrm{~m}$ depth. Whereas, instead of connecting the active dispersion curve with passive dispersion curve directly as the previous researchers usually do ${ }^{[7]}$, we applied a 1D SWT ${ }^{[11]}$, to calculate the phase velocity correspond to each frequency point and each segment of the survey line, then the phase velocity profile can be built to invert the Vs profile. The specific processing steps are as follows:

(1) Extracting the active phase velocity dispersion curve of fundamental-mode Rayleigh waves from each shot records by HRLRT.

(2) Calculating the cross-correlation for the ambient noise data recorded by each pair of seismic stations, with taking a negative derivative of the result, the empirical Green's function between these two stations can be retrieved. Then the passive phase velocity dispersion curve of fundamental-mode Rayleigh waves for each pair can be computed by automated FTAN method.

(3) Dividing the section of survey line into grids, whose horizontal axis corresponds to the line coordinates and vertical axis is varied frequencies corresponding to different penetration depth. With the dispersion curves of phase velocity for all paths obtained, the 1D SWT can be utilized to calculate the phase velocity for each grids, thus, the combined phase wave map is constructed.

(4) Inverting the obtained phase velocity dispersion curves at each grid for 1D shear velocity profiles and then assembling all these $1 \mathrm{D}$ profiles to construct a $2 \mathrm{D}$ shear velocity model.

\section{ApplicAtion to THE BALIKUN BASIN}

Experimental field surveys were conducted at the eastern Balikun Basin during July 2015 to August 2015. Through Observing long-time noises on a seismic array, the analysis of noise sources by beamforming method indicates that the most energetic sources come from southwest of the array, which is probably generated by the traffic noise from the main road. A linear layout from the foot of Balikun Mountain to the center of the basin was chosen along the direction of the noise approximately, which is cut off by the main road (C-1, C-2).

The active surveys and passive surveys were performed along line C-1 (5.4km) and C-2 (3.7km) (Fig. 1). 48 Reftek $125 \mathrm{~A}-01$ micro seismographs were used for the active surveys to record 48-channel data sets, with the receiver spacing of $10 \mathrm{~m}$, shot spacing of $30 \mathrm{~m}$ and sampling frequency of $250 \mathrm{~Hz}$. We used $2.5-\mathrm{Hz}$ vertical geophones as receivers and a vehicle with a sledgehammer as the source for the active surveys. Passive surveys also used Reftek 125A-01 to record noises for 5 hours at least, with the station spacing of $150 \mathrm{~m}$.

The active dispersion curves were extracted from each 48 channel data set by LRLRT, and the frequency range is about $6 \sim 30 \mathrm{~Hz}$. Although the noises is heterogeneous around the test site, the main noise energy is just along the survey line, so the empirical Green's function of Rayleigh waves can be retrieved from the noise cross-correlation. After selecting the function whose signal-to-noise ratio (SNR) is higher than 5, the FTAN method was used to calculate the phase velocity dispersion curves. In order to select the right dispersion curves of

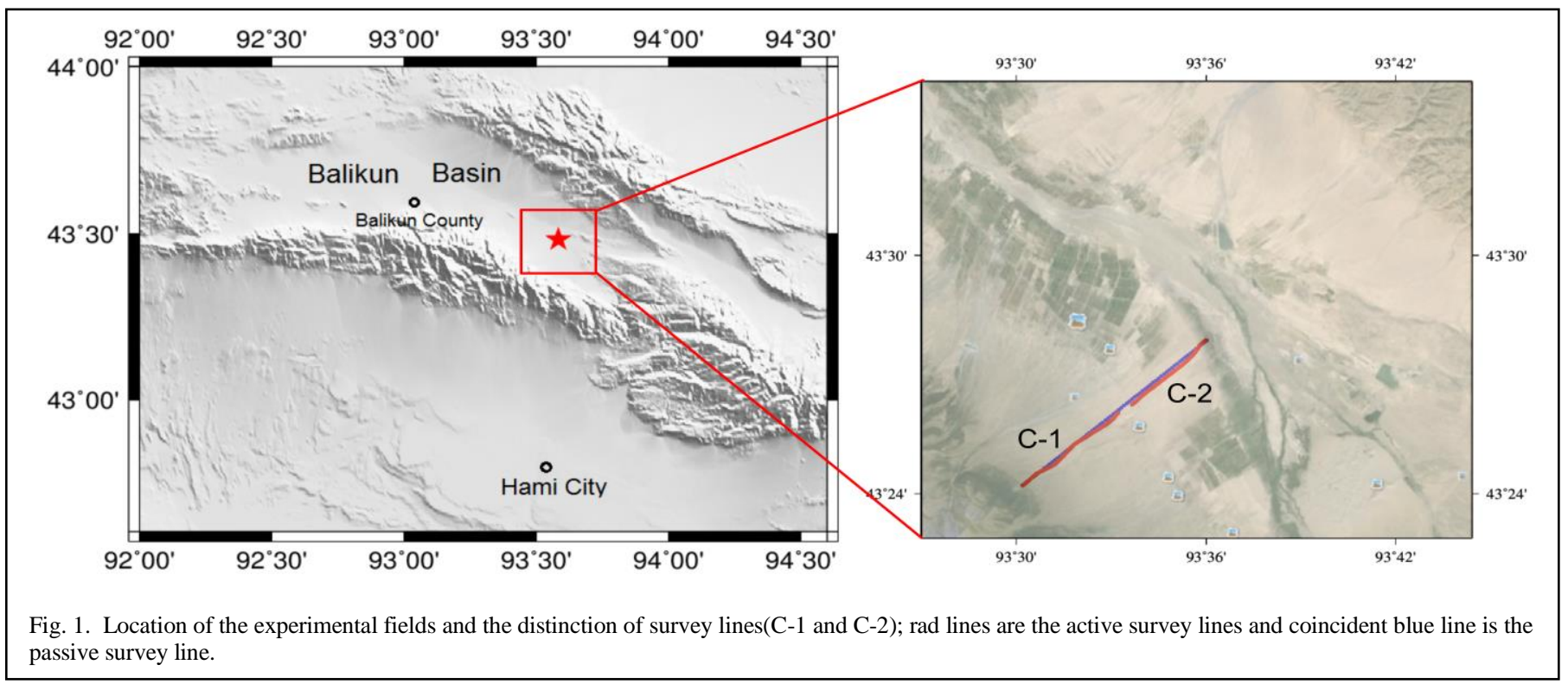




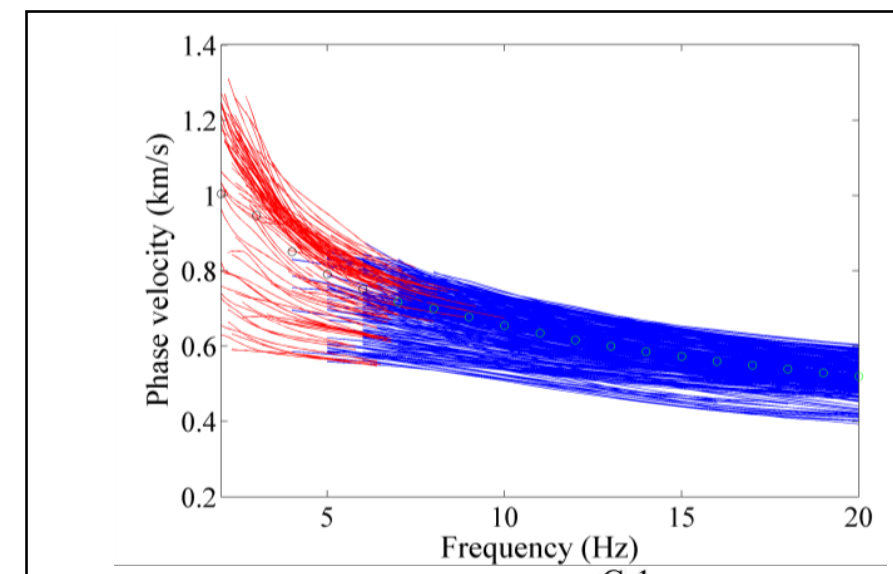

(c)

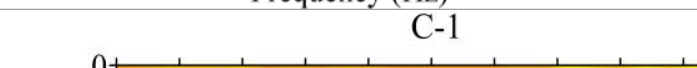

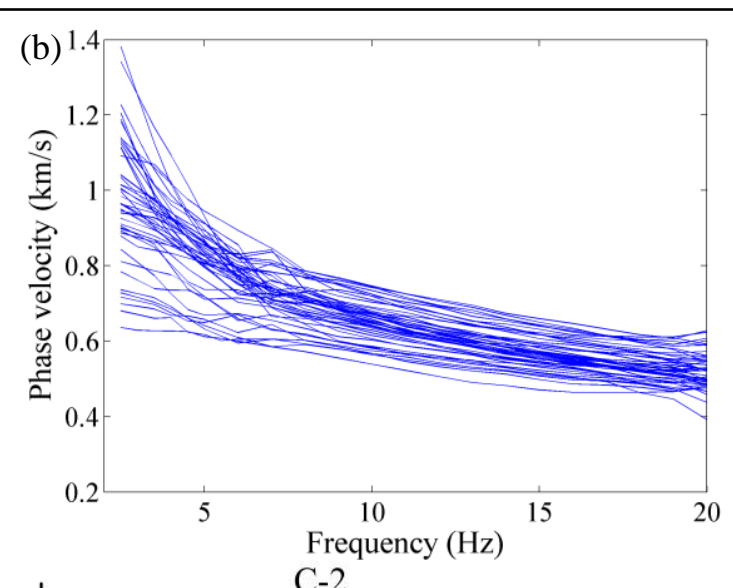

$\mathrm{C}-2$

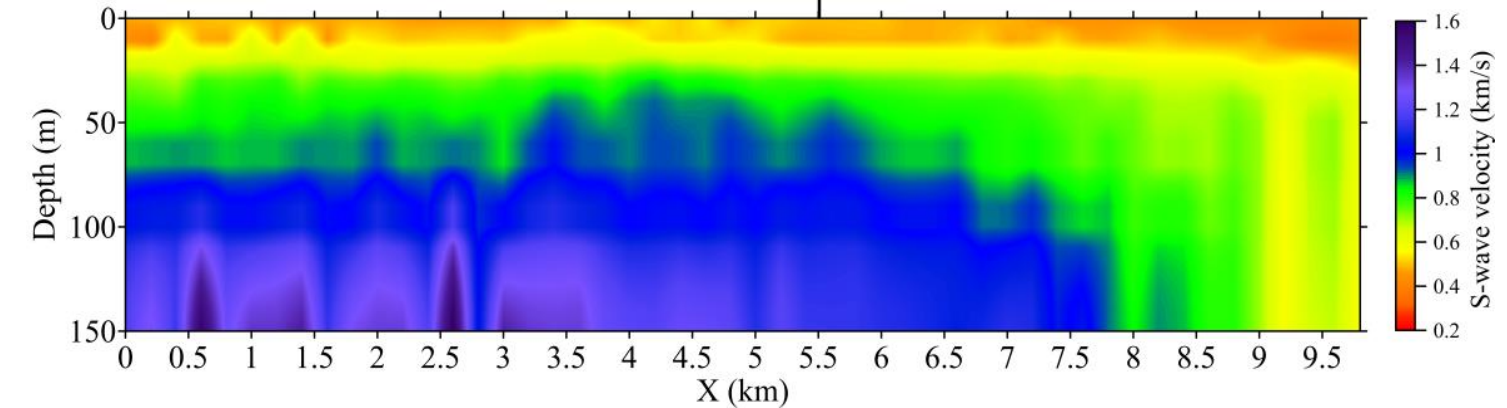

Fig. 2. (a) All the dispersion curves extracted from active surface wave (blue) and passive surface wave (red), the black circles mean the average of all the passive dispersion curves and the green circles mean the average of all the active dispersion curves. (b) The combined dispersion curves obtained through 1-D surface wave tomography for all segments. (c) Vs profile of line C-1 and C-2 inverted from the dispersion curves in (b).

fundamental-mode Rayleigh waves, an active dispersion curve from the nearest spreading was chosen as a reference. The frequency range of the passive dispersion curves is $2 \sim 10 \mathrm{~Hz}$.

Fig. 2a shows all the dispersion curves extracted from active and passive surface wave surveys. The 1D SWT was adopted to generate phase velocity maps at $2-20 \mathrm{~Hz}$ frequency range by using all the phase velocity dispersion curves from active and passive surveys. The dispersion curve of an active (or a passive) path is a comprehensive response to the phase wave velocity of near-surface materials between the first geophone to the last one (or the two stations). The line C-1 and C-2 were both divided into segments with $200 \mathrm{~m}$, which was determined by resolution checkboard. We assumed that each segment and frequency point have a constant phase velocity. Through using the 1-D SWT method, a new phase velocity dispersion-curve figure of $2 \sim 20 \mathrm{~Hz}$ has been obtained (Fig. 2b). The Vs profile of $\sim 150 \mathrm{~m}$ (Fig. $2 \mathrm{c}$ ) beneath the whole survey line was finally inverted by utilizing an iterative linearized least-square inversion scheme of surf $96^{[12]}$.

\section{Discussions}

The Vs profile of line C-1 and C-2 (Fig. 2c) shows that the investigation depth of active and passive surface wave combined method can reach $\sim 150 \mathrm{~m}$, which is much deeper than the investigation depth of active surface wave method (Fig. 3a). The Vs structure of shallow layers (<90m depth) in Fig. 2c is consistent with that in Fig. 3a approximately, with the former a little more smooth, because the grid size of Fig. $2 c(200 \mathrm{~m})$ is larger than that of Fig. 3a (30m). We should note that the Vs of each grid in Fig. 3a is not the real Vs of the grid, but the comprehensive Vs value of the underground mediums between the first geophone and the last one. While the SWT which inverts the phase velocity of each grid based on the travel paths and travel times is a better choose in theory. Whether the SWT method would reduce the horizontal resolution of the result and erase the small velocity anomalies or not, it still needs further research.

Fig. $3 \mathrm{~b}$ shows that the passive surface wave method can also achieve the goal of $\sim 150 \mathrm{~m}$ Vs structure, but it possesses low horizontal resolution at the shallow layers because of the lack of high-frequency contents which always reflect the information of shallow mediums. A composite Vs profile can also be constructed by splicing the two Vs profiles respectively obtained from active surface wave method (Fig. 3a) and passive surface wave method (Fig. 3b), but the suture places are unmatched. It implies that the inversion result of Vs structure at depth zone for passive survey may also be inaccurate due to the rather limited low frequency range of passive surface waves (Fig.3c).

In conclusion, the method combining active and passive surface waves by using SWT can achieve a greater depth of the Vs profile with ensuring the near-surface accuracy, which is a promising way for the exploration of $\sim 100 \mathrm{~m}$ depth.

The Vs profile (Fig.2c) shows that the mediums beneath the survey lines are layered and tilt towards the direction of the basin center, with the shear wave velocities varying from $\sim 200 \mathrm{~m} / \mathrm{s}$ near the surface to $\sim 1400 \mathrm{~m} / \mathrm{s}$ at $150 \mathrm{~m}$ depth. The drilling data near the line have also been acquired. These results suggest that the materials we have investigated are almost quaternary alluvium and alluvia sediments, which 
contain sandy soils and clays near the surface and a great deal of gravels in the deeper layers.

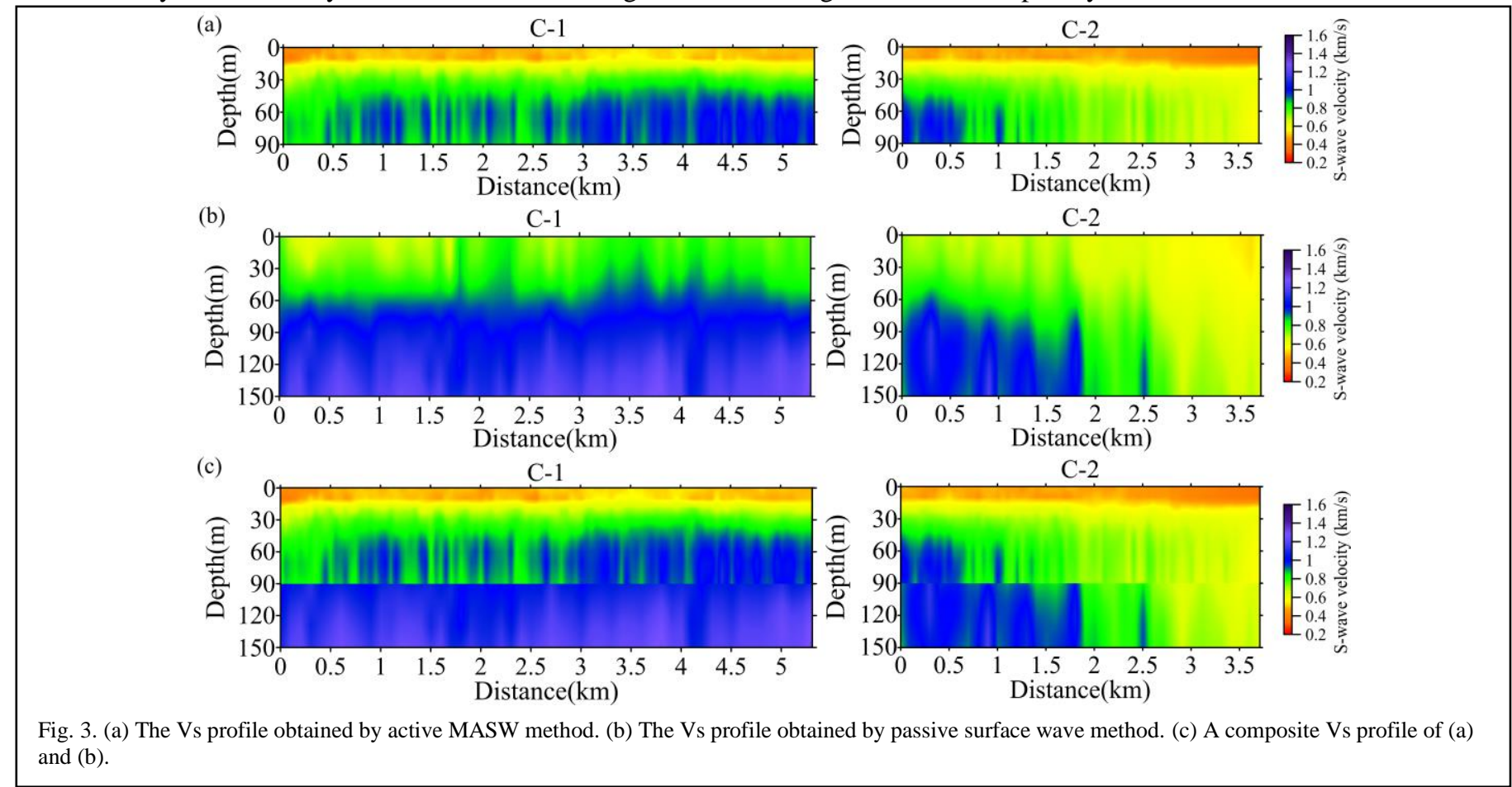

\section{CONCLUSIONS}

The method combining active and passive surface wave with 1D SWT is utilized to obtain the Vs profile of $\sim 150 \mathrm{~m}$ depth range beneath the Balikun Basin, which makes up the very limited exploration depth of traditional active MASW method. With the growing requirements for investigate depth on the engineering projects and resource prospection nowadays, the combined use of active and passive surface wave will be an alternative solution for a Vs investigation of $\sim 100 \mathrm{~m}$ depth. The combination methods of active and passive surface waves still need more research for credible and high-resolution Vs imaging.

\section{ACKNOWLEDGMENTS}

This research is supported by geological mapping project of Xinjiang. Thanks to our research group members for their work in data acquisition.

\section{REFERENCES}

[1] C.B. Park, R.D. Miller, and J. Xia, "Multichannel analysis of surface waves,” Geophysics, vol. 64, no. 3, pp. 800-808, May 1999.

[2] J. Xia, R.D. Miller, C.B. Park, "Estimation of near-surface shearwave velocity by inversion of Rayleigh waves," Geophysics, vol. 64, no. 3, pp. 691-700, May 1999.
[3] C.B., Park, R.D. Miller, "Roadside passive multi- channel analysis of surface waves (MASW)," J. Environ. Eng. Geophys., vol. 13, pp. 111, March 2008.

[4] G.D. Bensen, M.H. Ritzwoller, M.P. Barmin, et al., "Processing seismic ambient noise data to obtain reliable broad-band surface wave dispersion measurements," Geophys. J. Int., vol. 169, pp. 1239- 1260, June 2007.

[5] F.C. Lin, M.H. Ritzwoller, J. Townend, S. Bannister, M.K. Savage, "Ambient noise Rayleigh wave tomograhy of New Zealand," Geophys. J. Int., vol. 170, pp. 649-666, August 2007.

[6] M. Natale, C. Nunziata, G.F. Panza, "FTAN method for the detailed definition of VS in urban areas," 13th World Conference on Earthquake Engineering, Vancouver, B.C., Canada. Paper No. 2694, August 2004.

[7] C.B. Park, R.D. Miller, N. Ryden, J. Xia, J. Ivanov, "Combined Use of Active and Passive Surface Waves," J. Environ. Eng. Geophys., vol. 10, pp. 323-334, September 2005.

[8] Z. Wang, "Essentials and recent advances of the surface wave exploration technique," Geophysics and Geochemical Exploration, vol. 30, no.1, pp. 1-6. February 2006. (in Chinese)

[9] J. Wang, X. Sun, H. Wang, X. Xia, R. Cui, Application of Double source Surface Wave Prospecting in Coal Mined-out Area, Chinese Journal of Engineering Geophysics, vol.7, no. 4, pp. 403-407, August 2010. (in Chinese)

[10] Y. Luo, J. Xia, R.D. Miller, Y. Xu, J. Liu, Q. Liu, "Rayleigh-wave dispersive energy imaging using a high-resolution linear Radon transform," Pure Appl. Geophys., vol. 165, no. 5, pp. 903-922, May 2008. 